\title{
Unknot Diagrams Requiring a Quadratic Number of Reidemeister Moves to Untangle
}

\author{
Joel Hass · Tahl Nowik
}

Received: 20 November 2008 / Revised: 28 February 2009 / Accepted: 1 March 2009 /

Published online: 18 March 2009

(C) The Author(s) 2009. This article is published with open access at Springerlink.com

\begin{abstract}
Given any knot diagram $E$, we present a sequence of knot diagrams of the same knot type for which the minimum number of Reidemeister moves required to pass to $E$ is quadratic with respect to the number of crossings. These bounds apply both in $S^{2}$ and in $\mathbb{R}^{2}$.
\end{abstract}

Keywords Reidemister moves · Unknot

\section{Introduction}

In this paper we give a family of unknot diagrams $D_{n}$ with $D_{n}$ having $7 n-1$ crossings and with at least $2 n^{2}+3 n-2$ Reidemeister moves required to transform $D_{n}$ to the trivial diagram. These are the first examples for which a nonlinear lower bound has been established. We then use $D_{n}$ to construct for any knot diagram $E$ with $k$ crossings, a sequence of diagrams $E_{n}$ of the same knot type, having $k+7 n-1$ crossings, and requiring at least $2 n^{2}+3 n-2$ Reidemeister moves to transform to $E$.

A knot in $\mathbb{R}^{3}$ or $S^{3}$ is commonly represented by a knot diagram, a generic projection of the knot to a plane or 2-sphere. A diagram is an immersed oriented planar or spherical curve with finitely many double points, called crossings. Each crossing is marked to indicate a strand, called the overcrossing, that lies above the second strand,

The research of J. Hass was supported in part by NSF grant DMS-0306602.

J. Hass $(\bowtie)$

Department of Mathematics, University of California, Davis, CA 95616, USA

e-mail: hass@math.ucdavis.edu

T. Nowik

Department of Mathematics, Bar-Ilan University, Ramat-Gan 52900, Israel

e-mail: tahl@math.biu.ac.il 
Fig. 1 The three types of

Reidemeister moves

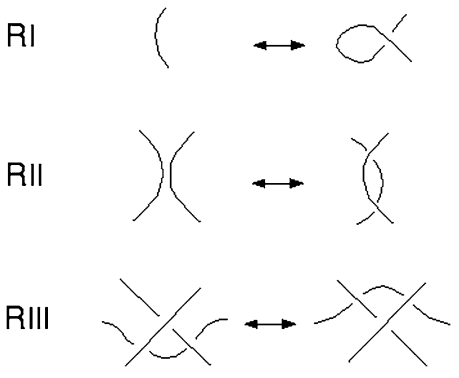

called the undercrossing. The original knot can be recovered, up to isotopy, by constructing a curve with the overcrossing arcs pushed slightly above the plane of the diagram and the remainder of the diagram lying in this plane.

Alexander and Briggs [1] and independently Reidemeister [9] showed that two diagrams of the same knot can be connected through a sequence of moves of three types, commonly called Reidemeister moves, shown in Fig. 1. The number of such moves required to connect two equivalent diagrams is difficult to estimate. An exponential upper bound is obtained in [4], where it is shown that there is a constant $C>1$ such that given an unknot diagram $D$ with $n$ crossings, no more than $C^{n}$ Reidemeister moves are required to transform $D$ to the trivial knot diagram.

We can get some lower bounds by looking at classical invariants of diagrams such as crossing numbers, writhes and winding numbers, since a single Reidemeister move changes these numbers by 0,1 , or 2 . However nontrivial lower bounds are difficult to obtain. This is a common situation in complexity theory. While upper bounds can be established by careful analysis of one procedure, lower bounds require somehow bounding from below the running time of all possible procedures. It is quite difficult to get lower bounds even for a particular pair of equivalent diagrams, as seen in $[2,3,7]$, and [8]. We note that examples are constructed in [6] that show that it may require exponentially many faces to construct a PL spanning disk for an unknotted polygon. However these examples can be transformed to the trivial diagram using only a linear number of Reidemeister moves.

Given two knot diagrams $D$ and $E$ of the same knot, we define the Reidemeister distance $d(D, E)$ between $D$ and $E$ to be the minimal number of Reidemeister moves required to pass from $D$ to $E$. One may consider this notion in either $S^{2}$ or $\mathbb{R}^{2}$, and our result will hold in both settings. Our main tool is an invariant of knot diagrams developed in [5] and used there to obtain new linear lower bounds on the Reidemeister distance.

\section{The Diagrams}

Let $U$ denote the trivial knot diagram. We will present a sequence $D_{n}$ of diagrams of the unknot, for which $d\left(D_{n}, U\right)$, the number of Reidemeister moves required to pass from $D_{n}$ to $U$, grows quadratically with respect to the number of crossings of $D_{n}$. More precisely, we prove: 
Fig. 2 The diagram $D_{n}$ for $n=4$

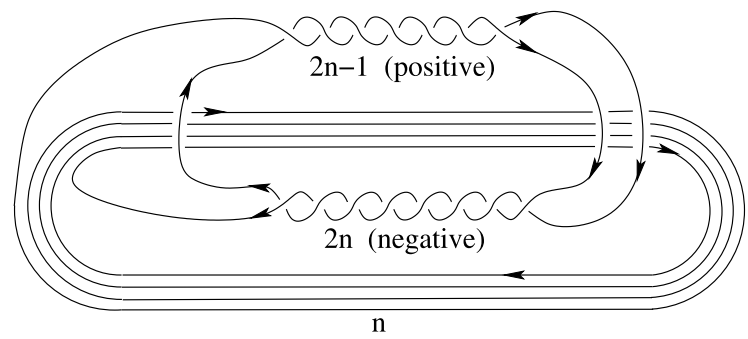

Theorem 2.1 In both $S^{2}$ and $\mathbb{R}^{2}$, the diagram $D_{n}$ of Fig. 2 which has $7 n-1$ crossings satisfies:

$$
2 n^{2}+3 n-2 \leq d\left(D_{n}, U\right) \leq 2 n^{2}+3 n .
$$

We will prove the lower bound in $S^{2}$ and the upper bound in $\mathbb{R}^{2}$, and it follows that both bounds hold in both settings. We recall the definition of the invariant of knot diagrams in $S^{2}$ introduced in [5]. We denote the set of all knot diagrams in $S^{2}$ by $\mathcal{D}$ and the set of all two component links in $\mathbb{R}^{3}$ by $\mathcal{L}$. Given a knot diagram $D \in \mathcal{D}$ and a crossing $a$ in $D$, define $D^{a} \in \mathcal{L}$ to be the two-component link obtained by smoothing the crossing $a$ (respecting the orientation of the strands). Given a knot diagram $D$, let $D_{+}$denote the set of all positive crossings in $D$ and $D_{-}$the set of all negative crossings. Given an invariant of two-component links $\phi: \mathcal{L} \rightarrow S$, where $S$ is any set, let $\mathbb{G}_{S}$ be the free abelian group with basis $\left\{X_{S}, Y_{S}\right\}_{S \in S}$. We then define the invariant $I_{\phi}: \mathcal{D} \rightarrow \mathbb{G}_{S}$ to be

$$
I_{\phi}(D)=\sum_{a \in D_{+}} X_{\phi\left(D^{a}\right)}+\sum_{a \in D_{-}} Y_{\phi\left(D^{a}\right)} .
$$

In this work $\phi$ is taken to be the linking number, $l k: \mathcal{L} \rightarrow \mathbb{Z}$, giving the invariant $I_{l k}: \mathcal{D} \rightarrow \mathbb{G}_{\mathbb{Z}}$. In [5] it is shown that the change in the value of $I_{l k}$ resulting from a Reidemeister move has one of the following forms:

1. For an RI move: $X_{0}$ or $Y_{0}$.

2. For an RII move: $X_{k}+Y_{k}$ or $X_{k}+Y_{k+1}$.

3. For an RIII move: $X_{k}-X_{k+1}$ or $Y_{k}-Y_{k+1}$.

Let $R$ be the set of elements in $\mathbb{G}_{\mathbb{Z}}$ of the form $X_{0}, Y_{0}, X_{k}+Y_{k}, X_{k}+Y_{k+1}, X_{k}-$ $X_{k+1}, Y_{k}-Y_{k+1}$, and their negatives. That is, $R$ is the set of all elements of $\mathbb{G}_{\mathbb{Z}}$ that may appear as the change in the value of $I_{l k}(D)$ as the result of performing a Reidemeister move on $D$. The set $R$ generates $\mathbb{G}_{\mathbb{Z}}$, and the length of an element of $\mathbb{G}_{\mathbb{Z}}$ with respect to this generating set is called its $R$-length. More precisely, the $R$ length of $x \in \mathbb{G}_{\mathbb{Z}}$ is the minimal $n$ for which one can write $x=\sum_{i=1}^{n} r_{i}$ with $r_{i} \in R$. Given two diagrams $D$ and $E$ of the same knot, the $R$-length of $I_{l k}(D)-I_{l k}(E)$ is a lower bound for $d(D, E)$ in $S^{2}$ and therefore also in $\mathbb{R}^{2}$. In particular, if $D$ is a diagram of the unknot, then since $I_{l k}(U)=0$, the $R$-length of $I_{l k}(D)$ gives a lower bound for $d(D, U)$. We use this procedure to obtain our lower bound for $d\left(D_{n}, U\right)$. 
Fig. $3 D_{n}$ after $2 n^{2}-n$ RIII moves and $2 n-1$ RII moves

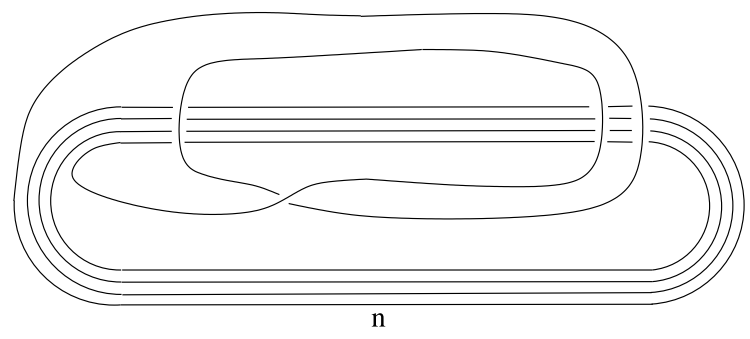

Fig. $4 D_{n}$ after an additional $n$ RII moves

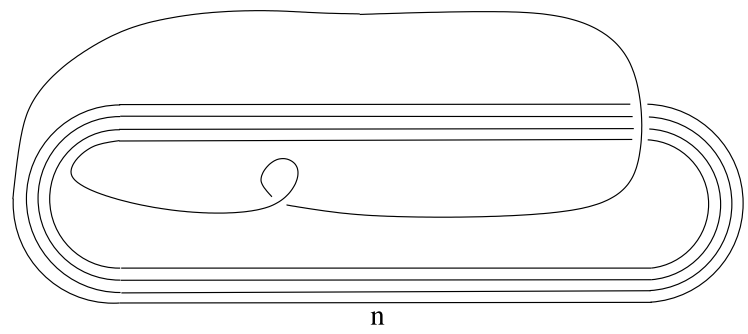

Proof of Theorem 2.1 A computation shows that

$$
I_{l k}\left(D_{n}\right)=n X_{n}+n X_{-n}+(2 n-1) X_{-1}+3 n Y_{0} .
$$

Indeed, each crossing in the top horizontal string of $2 n-1$ crossings contributes $X_{-1}$, each crossing in the bottom horizontal string of $2 n$ crossings contributes $Y_{0}$, each crossing in the left vertical line of $n$ crossings contributes $Y_{0}$, each crossing in the middle vertical line of $n$ crossings contributes $X_{n}$, and each crossing in the right vertical line of $n$ crossings contributes $X_{-n}$. Together this gives $n X_{n}+n X_{-n}+(2 n-$ 1) $X_{-1}+3 n Y_{0}$, and we denote this element of $\mathbb{G}_{\mathbb{Z}}$ by $v_{n}$.

We prove Theorem 2.1 by first showing that the $R$-length of $v_{n}$ is at least $2 n^{2}+$ $3 n-2$ and then demonstrating an explicit sequence of $2 n^{2}+3 n$ Reidemeister moves in $\mathbb{R}^{2}$ from $D_{n}$ to $U$.

Let $g: \mathbb{G}_{\mathbb{Z}} \rightarrow \mathbb{Z}$ be the homomorphism defined by $g\left(X_{k}\right)=1+|k|$ and $g\left(Y_{k}\right)=$ $-1-|k|$ for all $k$. Then $|g(r)| \leq 1$ for all $r \in R$, and $g\left(v_{n}\right)=2 n^{2}+3 n-2$. It follows that the $R$-length of $v_{n}$ is at least $2 n^{2}+3 n-2$.

It remains to demonstrate an explicit sequence of $2 n^{2}+3 n$ Reidemeister moves in $\mathbb{R}^{2}$, from $D_{n}$ to the trivial diagram. Start by sliding the top horizontal string of $2 n-1$ crossings in Fig. 2 in the clockwise direction, across the $n$ horizontal strands. This requires $n(2 n-1)$ RIII moves. Then cancel these $2 n-1$ positive crossings with $2 n-1$ of the negative crossings, now lying to the left of them, via $2 n-1$ RII moves, arriving at the diagram in Fig. 3. With $n$ additional RII moves we arrive at the diagram in Fig. 4. Finally, perform $n+1$ RI moves to get to the trivial diagram, resulting in a total of $2 n^{2}+3 n$ moves.

We conclude with an extension of Theorem 2.1 to general knots. 
Theorem 2.2 Let $E$ be any knot diagram with $k$ crossings. There exists a sequence of knot diagrams $E_{n}$ of the same knot with $E_{n}$ having $k+7 n-1$ crossings and with the property that

$$
2 n^{2}+3 n-2 \leq d\left(E_{n}, E\right) \leq 2 n^{2}+3 n .
$$

These inequalities hold both in $S^{2}$ and in $\mathbb{R}^{2}$.

Proof As noted in [5], the invariant $I_{l k}$ is additive with respect to the operation of connected sum of diagrams. Define $E_{n}=E \# D_{n}$, where we perform the connected sum operation, say at the right-hand most point of $D_{n}$ as it appears in Fig. 2. Then $I_{l k}\left(E_{n}\right)-I_{l k}(E)=I_{l k}\left(D_{n}\right)=v_{n}$, and so the same arguments as in the proof of Theorem 2.1 give the desired inequalities. For the upper bound, it suffices to notice that the explicit sequence of moves given in Theorem 2.1 leaves $E$ untouched.

Open Access This article is distributed under the terms of the Creative Commons Attribution Noncommercial License which permits any noncommercial use, distribution, and reproduction in any medium, provided the original author(s) and source are credited.

\section{References}

1. Alexander, J.W., Briggs, G.B.: On types of knotted curves. Ann. Math. 28, 562-586 (1926/1927)

2. Carter, J.S., Elhamdadi, M., Saito, M., Satoh, S.: A lower bound for the number of Reidemeister moves of type III. Topol. Its Appl. 153, 2788-2794 (2006)

3. Hagge, T.J.: Every Reidemeister move is needed for each knot type. Proc. Am. Math. Soc. 134(1), 295-301 (2006)

4. Hass, J., Lagarias, J.C.: The number of Reidemeister moves needed for unknotting. J. Am. Math. Soc. 14(2), 399-428 (2001)

5. Hass, J., Nowik, T.: Invariants of knot diagrams. Math. Ann. 342, 125-137 (2008)

6. Hass, J., Snoeyink, J., Thurston, W.P.: The size of spanning disks for polygonal curves. Discrete Comput. Geom. 29(1), 1-17 (2003)

7. Hayashi, C.: A lower bound for the number of Reidemeister moves for unknotting. J. Knot Theory Ramif. 15(3), 313-325 (2006)

8. Östlund, O.: Invariants of knot diagrams and relations among Reidemeister moves. J. Knot Theory Ramif. 10(8), 1215-1227 (2001)

9. Reidemeister, H.: Knoten und Gruppen. Abh. Math. Semin. Univ. Hamb. 5, 7-23 (1926) 